\title{
The Orientation of Cytochromes in Membrane Multilayers Prepared from Aerobically Grown Escherichia coli K12
}

\author{
By R. K. POOLE, ${ }^{1 *}$ H. BLUM, ${ }^{2}$ R. I. SCOTT, ${ }^{1}$ ANNETTE COLLINGE ${ }^{1}$ \\ AND T. OHNISHI ${ }^{2}$ \\ 1 Department of Microbiology, Queen Elizabeth College (University of London), \\ Campden Hill, London W8 7 AH \\ ${ }^{2}$ Johnson Research Foundation, Department of Biochemistry and Biophysics, University of \\ Pennsylvania, Philadelphia, Pennsylvania 19104, U.S.A.
}

(Received 2 January 1980)

\begin{abstract}
Centrifugation of membrane vesicles, prepared from ultrasonically disrupted Escherichia coli $\mathrm{K} 12$, on to a planar surface followed by slow, partial dehydration results in a high degree of parallel orientation of the membrane planes with respect to each other and the supporting surface. Rotation of such membrane multilayers about a single axis parallel with the membrane planes within the magnetic field of an electron paramagnetic resonance (e.p.r.) spectrometer allows the orientation of anisotropic paramagnetic centres to be deduced. Computer simulations of the angular dependence of cytochrome e.p.r. spectra show two, or perhaps three, cytochromes, well-oriented with respect to the membrane plane. A low-spin cytochrome is oriented with the normal to its haem plane lying in the membrane plane. One (or perhaps two) high-spin cytochrome(s) lies with its haem plane making an angle of $45^{\circ}$ with the membrane plane. The orientation of the low-spin cytochrome haem is thus the same as that of haems in $b$-type cytochromes and cytochrome oxidases of the $a$ type found in the mitochondria of higher animal and microbial cells and the bacterium Paracoccus denitrificans (Erecińska et al., 1979). The possible identity of this low-spin component as the terminal oxidase, cytochrome $o$, is discussed.
\end{abstract}

\section{INTRODUCTION}

Escherichia coli is capable of synthesizing at least nine different cytochromes (Haddock \& Jones, 1977), according to the growth conditions. In aerobically grown cells, cytochrome $o$ is the major terminal oxidase (Castor \& Chance, 1959), accepting electrons from a further $b$-type cytochrome (probably cytochrome $b_{556}$ or $b_{562}$; Poole \& Haddock, 1975) or from a quinone species (Downie \& Cox, 1978). The spectral (Shipp, 1972), potentiometric (Reid \& Ingledew, 1979), kinetic (Haddock et al., 1976; Poole et al., 1979 b) and ligand binding (Poole et al., 1979a,b) properties of some of these cytochromes have been studied, but virtually nothing is known about the spatial organization of the components within the membrane.

Information about the orientation and arrangement of membrane-bound enzymes, and particularly of their active sites (e.g. the haem group of cytochromes), is especially desirable for enzymes such as respiratory chain components that catalyse transmembrane (vectorial) reactions. It has been demonstrated that when mitochondrial membranes are centrifuged on to a planar surface and then partially dehydrated, they form multiple layers (membrane multilayers) in which the planes of the membranes become well-oriented, more-or-less parallel to each other and to the plane of the support (Erecińska et al., 1977; Blasie et al., 1978). Optical and electron paramagnetic resonance (e.p.r.) spectroscopy of such multilayers 
aligned at differing angles to the incident polarized light beam or magnetic field, respectively, allow the orientation of the chromophores with respect to the membrane plane to be deduced (Blasie et al., 1978). Thus, the planes of the haem groups in cytochromes $b, c_{1}$, $a$ and $a_{3}$ are oriented with their normals parallel to the membrane plane in mitochondria from animal tissues (Erecińska et al., 1978; Blum et al., 1978a) and from Tetrahymena pyriformis (Kilpatrick \& Erecińska, 1978, cited by Erecińska et al., 1979). The only bacterium whose cytochromes have been studied using this technique is Paracoccus denitrificans; its terminal oxidase, cytochrome $a a_{3}$, is oriented in a manner analogous to its mitochondrial counterpart (Erecińska et al., 1979).

In this paper, we describe e.p.r. studies of membrane multilayers prepared from aerobically grown $E$. coli and describe two orientations adopted by the haems of the cytochromes in the membrane. The possibility that the component whose orientation is like that of cytochrome oxidase in mitochondrial membranes is also the terminal oxidase (cytochrome $o$ ) in this system is discussed.

\section{METHODS}

Organism, growth conditions and preparation of membrane particles. Escherichia coli strain A1002 (K12 Y mel ilv lacI metE) was grown aerobically in a defined medium containing succinate as carbon source and supplemented with casein hydrolysate, exactly as described previously (Poole $e t$ al., 1979b). Cells were harvested in the exponential phase of growth, when $A_{420}(10 \mathrm{~mm}$ light path, $1 / 10$ dilution) was 0.3 to $0 \cdot 5$. They were washed, disrupted ultrasonically and fractionated to yield a membrane preparation essentially as described by Poole \& Haddock (1974). We prefer to avoid the use of the term 'electron transport particles' to describe these particles (Poole \& Haddock, 1974) since it may be taken to imply equivalence to particles prepared by alkaline treatment (Crane et al., 1956).

Preparation of oriented multilayers of membrane particles. Membrane particles stored at $-30^{\circ} \mathrm{C}$ were rapidly thawed, diluted about 15 -fold and homogenized with distilled water. They were centrifuged for $60 \mathrm{~min}$ at $23000 \mathrm{rev} \cdot \mathrm{min}^{-1}$ (70000 $\mathrm{g}$ at $r_{\mathrm{av}}$ ) on to a circular sheet of Mylar (a plastic film about $0.13 \mathrm{~mm}$ thick). The plane of the Mylar was supported at a normal to the centrifugal force using a specially constructed Perspex insert for the tubes of the swinging bucket SW25.1 rotor of a Beckman L5-50 ultracentrifuge. The Mylar sheet had previously been washed with detergent and water and then coated with a film of collodion by dipping in a $1 \%(\mathrm{w} / \mathrm{v})$ solution in ethanol/ether $(75: 25, \mathrm{v} / \mathrm{v})$. After centrifugation, the Mylar sheet and the adhering pellet of particles $(0.5$ to $1.0 \mathrm{~cm}$ diam., 0.5 to $1.0 \mathrm{~mm}$ thick) was drained of excess liquid and slowly, partially dehydrated by incubating in an atmosphere of $90 \%$ relative humidity at $4{ }^{\circ} \mathrm{C}$ for 48 to $72 \mathrm{~h}$. Membranes in such multilayers are oxidized as demonstrated by the e.p.r. spectra (see Results).

Electron paramagnetic resonance (e.p.r.) studies. The hydrated multilayer on its Mylar support was sliced into rectangular pieces, each approx. $2 \times 10 \mathrm{~mm}$. Usually two or three of these pieces were slipped into a standard e.p.r. tube. The top of the tube was sealed and fitted with a pointer that was parallel to the plane of the Mylar sheets. The tube was mounted in a tubular holder fitted with a protractor, so that the angle of the Mylar in the magnetic field could be read. The experimental arrangement is shown in Fig. 1. Field angles given are those between the direction of the magnetic field and the normal to the Mylar film; that is, the angle is given as $90^{\circ}$ when the plane of the Mylar is parallel to the incident magnetic field. Referring to Fig. 1 of Blum et al. (1978a), the angle quoted is shown as $\omega$. E.p.r. spectra were taken on a Varian E109 spectrometer. Details of sample cooling and temperature control have been described by Blum et al. (1978a).

Data were analysed with the aid of a computer program (Blum et al., 1978b). The e.p.r. spectrum of membrane-bound chromophores in oriented membrane multilayers can be simulated by a model which assumes a fixed orientation of the paramagnetic centre relative to the membrane normal and no order with respect to rotation about the membrane normal. The program models mosaic spread (wobble of the chromophore in the direction of the membrane normal) using a Gaussian distribution of chromophore orientations (see Fig. 1 in Blum et al., 1978b).

Protein determinations. An estimate of the particle protein present on Mylar films was obtained by treating small slices (about 2 to $3 \mathrm{~mm}^{2}$ ) of the Mylar and attached particles with $0.5 \mathrm{M}-\mathrm{NaOH}$ for 7 min at 95 to $100^{\circ} \mathrm{C}$. The digested protein was assayed by Lowry's method using dry bovine plasma albumin as standard. The multilayers described here contained of 22 to $34 \mu \mathrm{g}$ protein $\mathrm{mm}^{-2}$.

Electron microscopy. Partially hydrated, oriented preparations on their Mylar films were fixed in $1.5 \%$ $(\mathrm{w} / \mathrm{v})$ glutaraldehyde in $0.1 \mathrm{M}$-sodium cacodylate buffer for $60 \mathrm{~min}$, then rinsed in similar buffer and postfixed in $1 \%(\mathrm{w} / \mathrm{v}) \mathrm{OsO}_{4}$ also made up in this buffer. Dehydration by passage through graded water/ethanol 


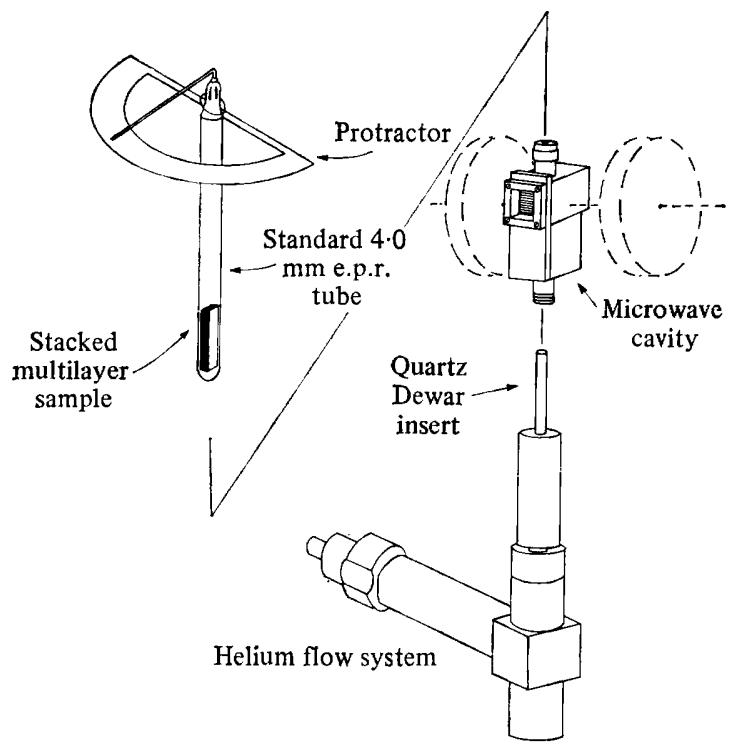

Fig. 1. Schematic diagram of the device for orientation and rotation of the multilayer samples. The standard e.p.r. tube contained two or more slices (approx. $2 \times 10 \mathrm{~mm}$ each) of the multilayer preparation, carefully stacked in parallel, and was sealed and capped with a pointer. The entire assembly was inserted in a special large-bore quartz Dewar in an Air Products LTD-3-110 flowing helium cryostat. The dashed lines indicate the position of the electromagnet on either side of the rectangular microwave cavity.

mixtures was followed be embedding in Spurr's resin. Sections (about $80 \mathrm{~nm}$ thick) were cut at a normal to the Mylar plane.

For examination of unoriented membrane particles, a portion $(0.3 \mathrm{ml})$ of particle suspension $\left(13 \mathrm{mg} \mathrm{m} l^{-1}\right)$ was mixed on a glass slide with an equal volume of just-molten $3 \%(\mathrm{w} / \mathrm{v})$ purified agar (Difco) dissolved in disruption buffer (50 mM-Tris/HCl, $2 \mathrm{mM}-\mathrm{MgCl}_{2}, 1 \mathrm{~mm}$-EGTA, $\mathrm{pH} 7 \cdot 4$ ). When set, small cubes of the agar (about $1 \mathrm{~mm}^{3}$ ) were cut and treated exactly as described above, except that they were embedded in Taab resin (Taab, Reading, Berks.).

\section{RESULTS}

\section{Electron microscopy of membrane multilayers}

Electron micrographs of the membrane preparation centrifuged on to a Mylar surface are presented in Fig. 2. Examination of the partially hydrated preparation at an intermediate depth in the multilayer (Fig. $2 a$ ) shows the membranes to be laminar structures with their planes substantially oriented in parallel with the Mylar plane. At the edge of the multilayer (Fig. $2 b$ ), orientation is inferior and many membrane particles retain a vesicular appearance, though somewhat flattened, and with their longer axes parallel to the edge of the multilayer.

It is difficult to assess the degree of orientation in our preparations solely from morphological analysis. It must be assumed that no loss of orientation occurs during the preparative procedures following fixation and that the sections are cut at a true perpendicular to the Mylar plane. Furthermore, the Mylar film becomes detached during sectioning (Fig. $2 b$ ). Nevertheless, an estimate of the unoriented fraction can be obtained by assuming that the vesicles are spherical and, on centrifugation and dehydration, flatten to give two more or less parallel layers with curved ends. Larger vesicles will have relatively more area in the multilayer form than will smaller vesicles. If the surface area of a vesicle of radius $R$ is taken to be $4 \pi R^{2}$, the curved portion of the surface on flattening will be approx. $2 \pi R \times \pi r$, where $r$ is the membrane thickness (Blum et al., 1978a). We take as our estimate of vesicle diameter a value of $0 \cdot 1 \mu \mathrm{m}$. This was obtained from measurements of vesicles seen in electron micro- 

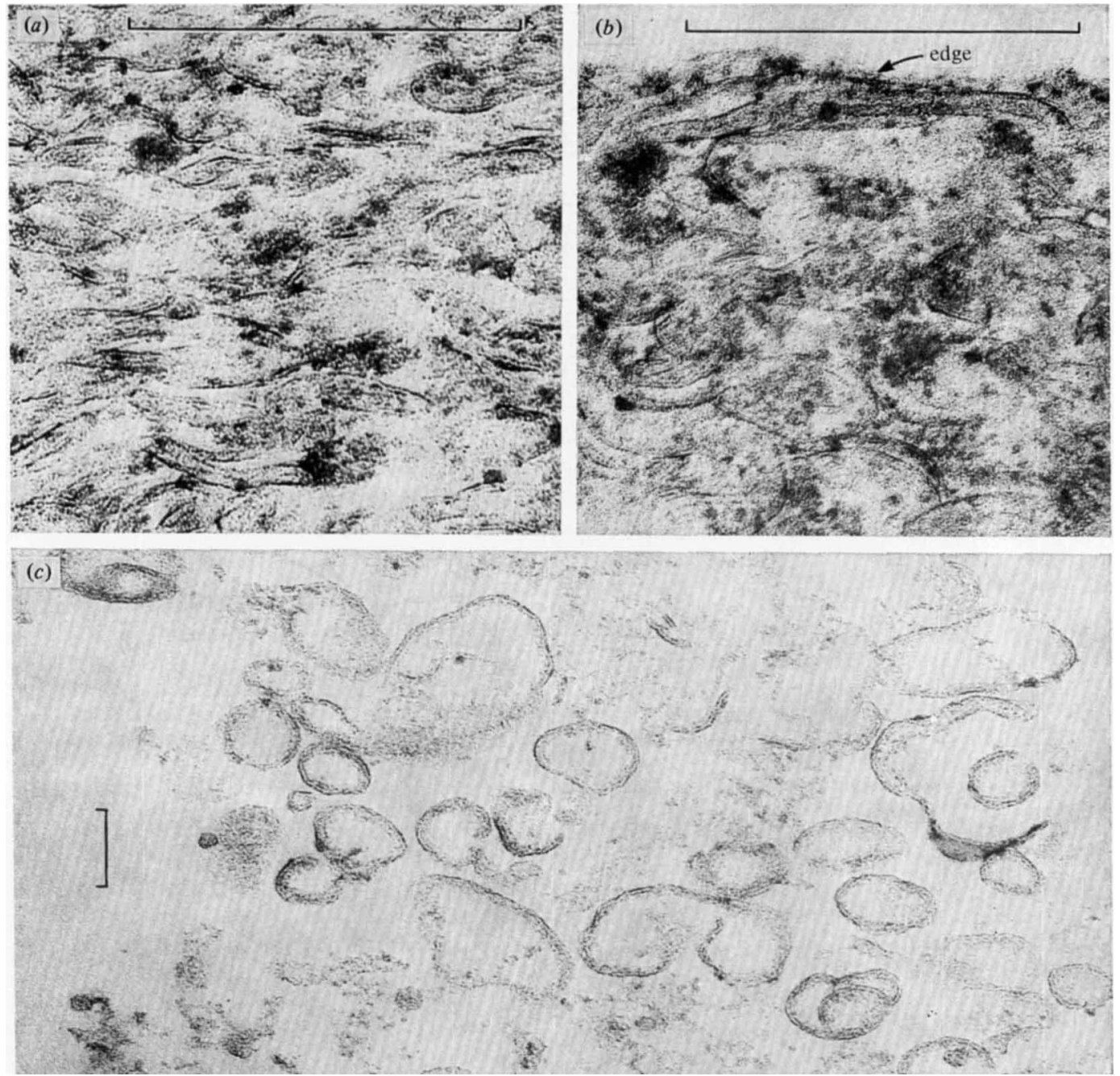

Fig. 2. Electron micrographs of membrane particles from $E$. coli. (a) and (b) show membranes centrifuged on to Mylar sheets. The sections were cut in a plane perpendicular to the Mylar plane (parallel with the centrifugal field): (a) portion at an intermediate depth in the multilayer; $(b)$ the multilayer edge. The Mylar film became detached from the multilayer during sectioning. In (a) and $(b)$, the bar markers represent $0.5 \mu \mathrm{m}$ and also indicate the plane of the Mylar sheet. In $(c)$, the suspension of unoriented particles was mixed with agar before fixation and further processing; the bar marker represents $0 \cdot 1 \mu \mathrm{m}$.

graphs of unoriented particles in suspension (Fig. $2 c$ ): the mean diameter was $0 \cdot 1 \mu \mathrm{m} \pm$ S.D. $0.037 \mu \mathrm{m}$ ( 79 measurements). This estimate is in agreement with the range of values given by other authors for particles prepared from $E$. coli using either the French press or sonication $(0.04$ to $0.11 \mu \mathrm{m}$; Altendorf \& Staehelin, 1974). Thus, the minimal unoriented fraction $[(\pi / 2)(r / R)]$ is estimated to be $22 \%$, if the membrane thickness is $7 \mathrm{~nm}$ (Kaback, 1971).

\section{Signals from low-spin cytochromes detectable in oxidized membrane multilayers}

The electrons with unpaired spins that give rise to the e.p.r. spectrum are primarily localized in the $d$-orbitals of the haem iron. Transition metal ions such as iron may have more than one unpaired electron. When maximal pairing of electrons occurs in the shells of lower energy levels (i.e. when the pairing energy is less than the field splitting energy) the 


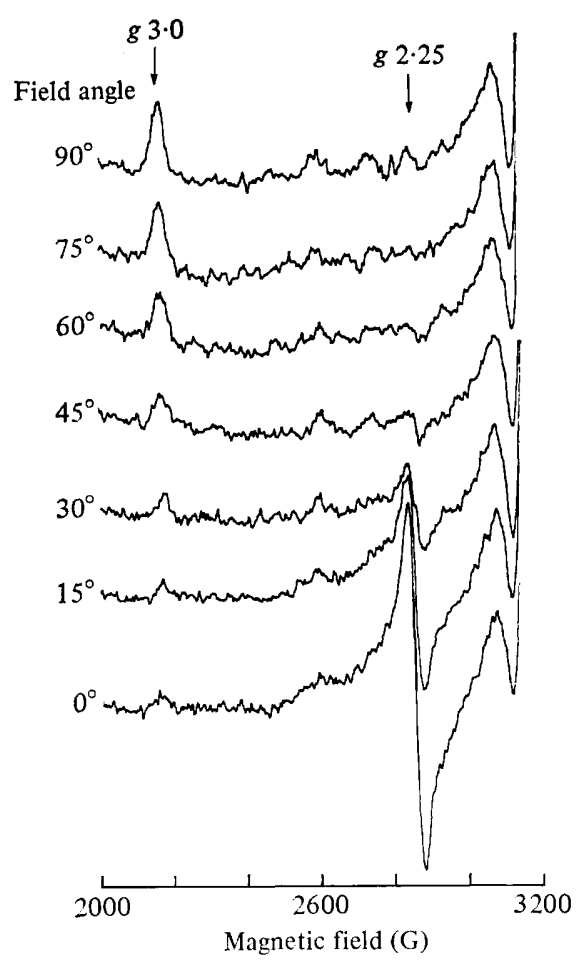

Fig. 3
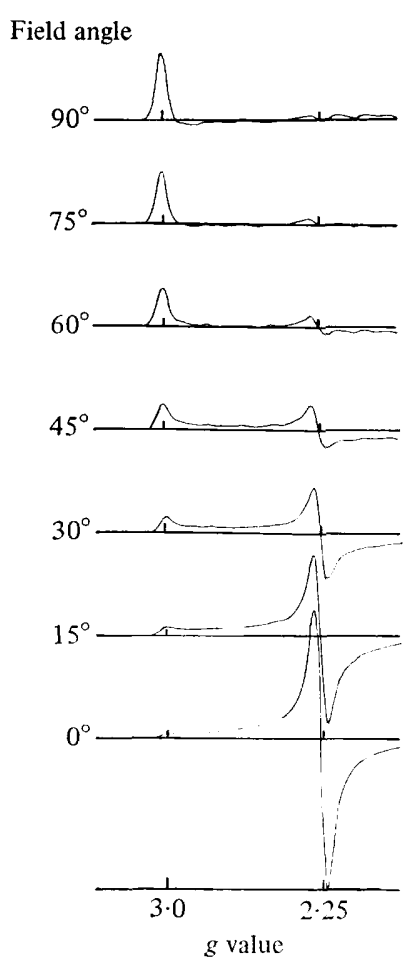

Fig. 4

Fig. 3. E.p.r. derivative spectra of air-oxidized membrane multilayers at $11 \mathrm{~K}$ in the field region between 2000 and $3200 \mathrm{G}$. Field angle is defined as the angle between the normal to the membranes (strictly, the Mylar support) and the magnetic field. E.p.r. conditions were as follows: incident microwave power, $10 \mathrm{~mW}$ at $9.06 \mathrm{GHz}$; modulation amplitude, $20 \mathrm{G}$ at $100 \mathrm{kHz}$; scan rate, $1000 \mathrm{G} \mathrm{m^{-1 }}$; time constant $0 \cdot 128 \mathrm{~s}$.

Fig. 4. Computer simulation of the angular dependence of the low-field portion of the e.p.r. spectra of oxidized membrane multilayers. A disorder mosaic spread angle of $35^{\circ}$ was assumed. The line-width was assumed to be constant at $30 \mathrm{G}$ and line shape to be Gaussian. The $g_{\mathrm{y}}$ axis is assumed to be along the membrane normal and the $g_{\mathrm{x}}$ and $g_{\mathrm{z}}$ axes are in the membrane plane as shown in Fig. 6.

complex is said to be low-spin. Alternatively, a high-spin complex arises when the splitting energy is small and the electrons are largely unpaired. In the description of e.p.r. results that follows, the subscripts $\mathrm{x}, \mathrm{y}$ and $\mathrm{z}$ denote those $g$ values attributed to the principal molecular axes of the paramagnetic centre, relative to the magnetic field direction. [For an elementary treatment of these topics, see Knowles et al. (1976).]

Figure 3 shows e.p.r. spectra at $11 \mathrm{~K}$ of membrane multilayers prepared from aerobically grown E. coli. As the multilayer was rotated about an axis parallel to the long axis of the Mylar (Fig. 1) in the magnetic field, the intensity of signals from paramagnetic centres varied. At a field angle of $90^{\circ}$ (i.e. when the Mylar plane was parallel to the magnetic field), a signal with a $g$ value of 3.0 was prominent, characteristic of a low-spin oxidized haem (Van Gelder et al., 1967). This signal is attributed to a principal $g$ value $\left(g_{\mathrm{z}}\right)$ of an anisotropic centre. A second principal signal $\left(g_{\mathrm{y}}\right)$ attributable to the centre is seen at $g=2 \cdot 25$, but only when the field angle approached $0^{\circ}$ (i.e. when the Mylar plane was perpendicular to the magnetic field). The accompanying third $g$ value signal $\left(g_{\mathrm{x}}\right)$, usually found around $g=1 \cdot 25$, was too broad to be seen. The angular dependence of these $g$ signals is more clearly seen when the signal amplitudes (in arbitrary units) are presented as a function of 


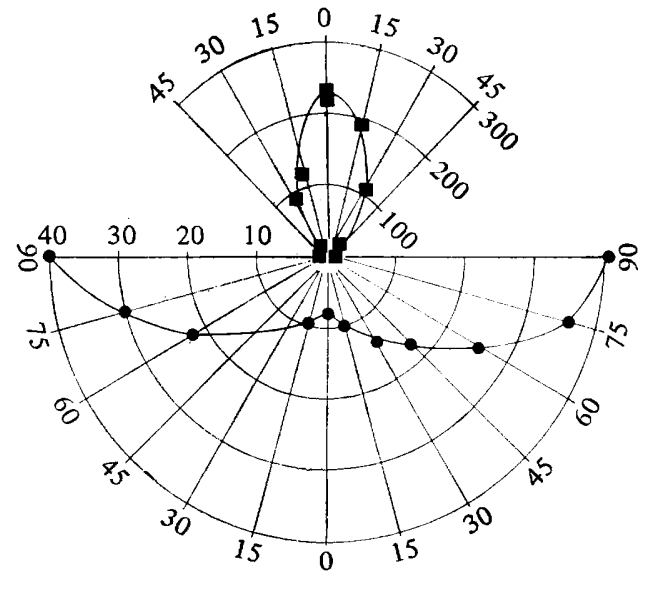

Field angle (degrees from multilayer normal)

Fig. 5

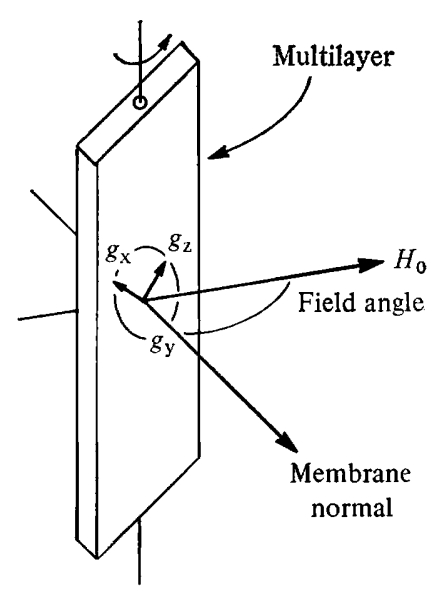

Fig. 6

Fig. 5. Polar plots showing the angular dependence of the e.p.r.-detectable signals in oxidized membrane multilayers. Amplitudes of the signals at $g_{\mathrm{z}}=3.0(\mathbf{O})$ and $g_{\mathrm{y}}=2.25(\boldsymbol{\mathbb { C }})$, in arbitrary units, are plotted along the radii as a function of field angle. The amplitudes measured during rotation of the multilayer through $180^{\circ}$ are shown.

Fig. 6. Diagram to illustrate the proposed spatial relationships between the membrane multilayer, its normal and the principal $g$ axes of the cytochrome seen in the e.p.r. spectra of Fig. 3 . The $g_{y}$ axis is shown lying along the membrane normal that makes the field angle indicated (varied by rotating about the vertical axis) with the magnetic field $\left(H_{0}\right)$. The axes $g_{\mathrm{z}}$ and $g_{\mathrm{x}}$ lie in the plane of the multilayer each at $90^{\circ}$ to the other and to $g_{\mathrm{y}}$.

field angle in polar plots (Fig. 5). These show that the $g=3.0$ signal is maximal at a field angle of $90^{\circ}$, decreases to a minimum at $0^{\circ}$ and, as rotation is continued through $180^{\circ}$ from the original position, increases again to a maximum. The converse is true for the $g=2 \cdot 25$ signal, except that the signal intensity was too low to measure and plot at field angles between $45^{\circ}$ and $90^{\circ}$.

We have analysed the data presented above with the assistance of a computer program. Given the principal $g$ values, three line-width parameters, the mosaic spread and the assumed orientation of the paramagnetic centre, a simulation of the e.p.r. spectrum was computed. Comparison of the simulation (Fig. 4) and the data (Fig. 3) shows that an excellent simulation is obtained when the $g_{\mathrm{y}}$ axis of the haem is assumed to be along the membrane normal and the $g_{\mathrm{z}}$ axis lies in the membrane plane. The $g_{\mathrm{x}}$ axis must also lie in the multilayer plane at right angles to $g_{\mathrm{y}}$ and $g_{\mathrm{z}}$ (Fig. 6).

\section{Signals from high-spin cytochromes detectable in oxidized membrane multilayers}

Figure 7 shows the e.p.r. spectra of oxidized multilayers in the magnetic field region between about 900 and $1600 \mathrm{G}$. We attribute the signals near $g=6.0$ to two high-spin cytochromes. The major component has $g_{\mathrm{x}}=g_{\mathrm{y}}=6 \cdot 0$ (with $g_{\mathrm{z}}=2$ and not observed) whilst the minor component has $g_{x}=6.3$ and $g_{y}=5.7$ (with $g_{z}$ not observed). The prominent isotropic signal at $g=4 \cdot 3$, whose intensity is independent of field angle, is thought to be ferric iron in a field of low symmetry, no longer specifically associated with protein(Blumberg, 1967).

The signal at $g=6 \cdot 0$, arising from a centre of axial symmetry, does not vary with field angle (Fig. 7), but this does not necessarily imply that it is not well-oriented in the magnetic field. It is possible for the $g_{\mathrm{x}}$ and $g_{\mathrm{y}}$ signals to vary separately such that the sum of their magnitudes remains relatively constant. The rhombic minor signal can be used as an aid in elucidating the orientation of the axial signal since the $g_{\mathrm{x}}$ and $g_{\mathrm{y}}$ signals are sufficiently 


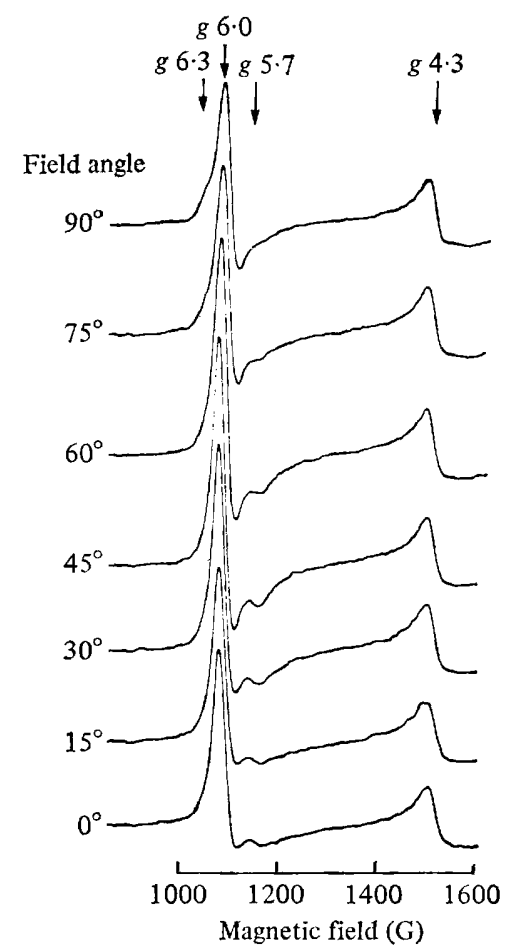

Fig. 7

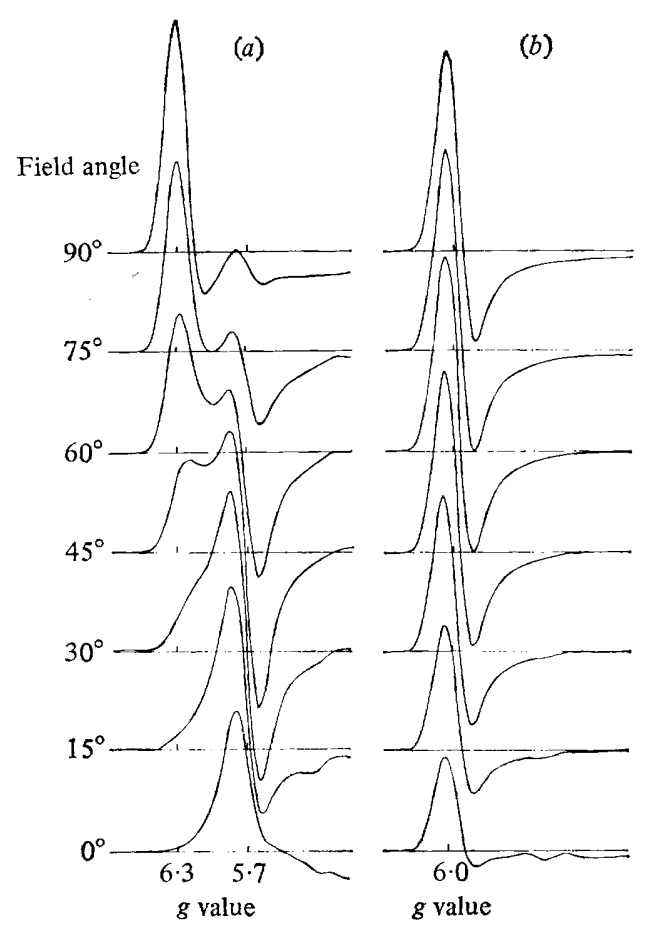

Fig. 8

Fig. 7. E.p.r. derivative spectra of air-oxidized membrane multilayers at $11 \mathrm{~K}$ in the field region between about 900 and $1600 \mathrm{G}$. Conditions were those described for Fig. 3 except that the receiver sensitivity was $0 \cdot 1$ of that of Fig. 3.

Fig. 8. Computer simulations of the angular dependence of the e.p.r. signals from high-spin cytochrome(s). Simulations of the $g_{\mathrm{x}}=6.3$ and $g_{\mathrm{y}}=5.7(\mathrm{a})$ and the $g_{\mathrm{x}}=g_{\mathrm{y}}=6.0(\mathrm{~b})$ signals are shown. The disorder mosaic spread angle was assumed to be $30^{\circ}$. The line-width was taken to be constant at $30 \mathrm{G}$. Orientation of the cytochrome(s) was assumed to be with the $g_{\mathrm{z}}$ axis at $45^{\circ}$ to the membrane normal and the $g_{\mathbf{x}}$ axis in the membrane plane.

separated to be seen as individual signals. Inspection of Fig. 7 shows that the $g_{\mathrm{x}}$ signal at $g=6.3$ is maximal at a field angle of $90^{\circ}$, while the $g_{y}$ at $g=5.7$ is maximal around $30^{\circ}$.

The computer simulations shown in Fig. 8(a) fit these data well. Here, the $g_{\mathrm{x}}$ axis is assumed to lie in the membrane plane and the $g_{y}$ axis is assumed to lie $45^{\circ}$ from the membrane plane. This would restrict the $g_{z}$ axis to lie at $45^{\circ}$ from the plane also. In Fig. $8(b)$, the same orientation is assumed for the major $(g=6)$ signal with good results. Note that the simulated curves fit the data well with respect to the fraction of the $g=6 \cdot 0$ signal that lies below the baseline.

\section{DISCUSSION}

The results presented here demonstrate that the techniques devised for preparing oriented membrane multilayers from various biological structures, such as mitochondria, reconstituted cytochrome oxidase vesicles and chromatophores (see references in Introduction; also Tiede et al., 1978; Salerno et al., 1979), are applicable also to E. coli membranes prepared by ultrasonic disruption. Indeed, the high degree of orientation of cytochromes shown in the present paper (see especially Fig. 3,5) compares very favourably with that obtained in multilayers from Paracoccus denitrificans (Erecińska et al., 1979), the only other bacterium to have been studied by these methods. In view of the effect of the size of the membrane vesicles used on the degree of disorder in the multilayers, it would be of interest 


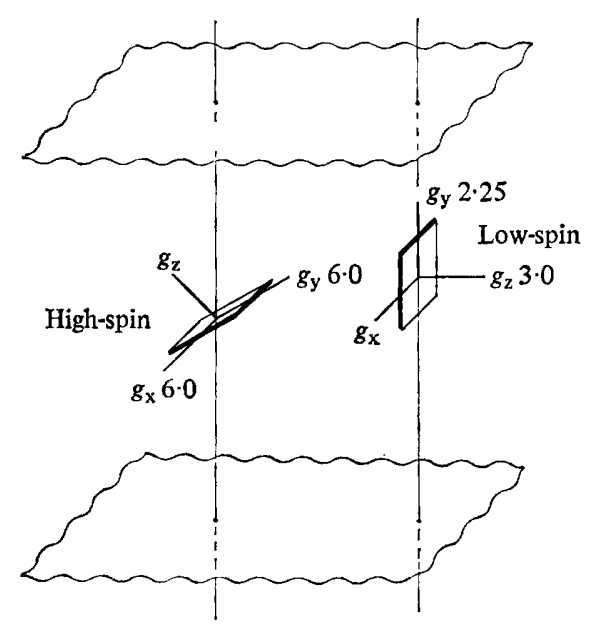

Fig. 9. Diagram to illustrate the proposed orientation of two cytochrome haems in the cytoplasmic membrane of $E$. coli. The plane of the membrane is horizontal (almost perpendicular to the page); the upper and lower faces of the membrane are shown. The orientations of haems in a high-spin (left) and low-spin (right) cytochrome are shown and are consistent with the data presented in this paper.

to extend this work to the large vesicles ( $1 \cdot 1$ to $1.4 \mu \mathrm{m}$ diam.) prepared by the method of Kaback (1971).

Our e.p.r. spectra disclose a total of three cytochromes. However, experience with other high-spin cytochromes (Blum et al., 1978c, d; Peisach et al., 1971) shows that multiple conformations are possible for a single cytochrome. These various conformations may not have functional significance. At the present time we can, therefore, only conclude that there are at least two distinct cytochromes present in these multilayers, a low-spin cytochrome and a high-spin cytochrome. Spectrophotometric studies and numerical analysis reveal at least five different cytochrome bands in $E$. coli, tentatively assigned to two $c$-type and three $b$-type cytochromes (Shipp, 1972). It is generally agreed that the cytochromes $c$ are 'soluble' and substantially lost from membrane preparations (Haddock \& Jones, 1977).

Evidence for three $b$-type cytochromes also come from potentiometric data (Hendler et al., 1975; Reid \& Ingledew, 1979), although Pudek \& Bragg (1976) resolved only two major $b$-type cytochromes. Concentrations of cytochromes $a_{1}$ and $d$ are low or undetectable in aerobically grown cells in the exponential phase of growth (Castor \& Chance, 1959; Poole et al., 1979b). The two or three cytochromes described here, therefore, are most likely to be cytochromes of the $b$-type. It should be noted that such cytochromes tend to have very broad e.p.r. spectra and their spectra may have signal-to-noise ratios that preclude their observation.

On the basis of the data presented here, we are unable to ascribe the e.p.r.-detectable signals to the specific cytochromes described in optical spectra. Extensive characterization, especially of potentiometric properties, and temperature and power saturation characteristics of their e.p.r. spectra will be required. The high-spin cytochrome at $g=6.0$ has a midpoint potential in unoriented particles at $\mathrm{pH} 7 \mathrm{of}+150 \mathrm{mV}$, with $n=1$ (R. K. Poole, $\mathrm{H}$. Blum \& T. Ohnishi, unpublished results), and thus does not directly correspond to the b-type cytochromes observed by Reid \& Ingledew (1979) or Hendler et al. (1975) in other strains of $E$. coli grown under somewhat different growth conditions.

At this time, our knowledge of the relationship between the principal $g$ axes and the orientation of porphyrin rings (e.g. haem) rests mainly on the e.p.r. and X-ray data on horse-heart cytochrome $c$ and myoglobin derivatives (for references, see Blum et al., 1978a). By analogy with these results, the $g_{\mathrm{z}}$ axes of the cytochromes studied here are shown to be 
close to the haem normal in the summary diagram of Fig. 9. Based on our deductions of the orientations of the principal $g$ axes, the haem plane of the low-spin cytochrome is thus shown as perpendicular to the membrane plane whilst that of the high-spin cytochrome is shown inclined at $45^{\circ}$ to the membrane plane.

The low-spin cytochrome exhibits certain features that suggest it may be the terminal oxidase in these cells, cytochrome $o$ (Castor \& Chance, 1959). Using photoselection and circular dichroism techniques similar to those employed by Junge \& Devault (1975) for the study of mitochondrial cytochrome $a_{3}$, Garland (1979) and Garland et al. (1979) were unable to detect dichroism of membrane-bound cytochrome $o$ of $E$. coli after laser-flash photolysis of its CO complex. This absence of dichroism was explained by proposing that the haem plane of the cytochrome was perpendicular to the membrane and that the chromophore rotated rapidly about an axis also perpendicular to the membrane with a rotational relaxation time $(\tau)$ of $<100 \mu \mathrm{s}$. This axis is therefore consistent with that deduced for the low-spin component in the present paper (Fig. 9). It is also of interest that in all of the systems investigated using the membrane multilayer technique, the haems of terminal oxidases have been found to be oriented perpendicular to the planes of the multilayers. These results are summarized by Erecińska et al. (1979) and include data for the $a$-type oxidases in two micro-organisms, Paracoccus denitrificans and Tetrahymena pyriformis. It is tempting to speculate that the function of cytochrome oxidases requires that the oxidase haems be oriented in a characteristic fashion with respect to the membrane plane.

The orientation of the high-spin cytochrome is also shown diagramatically in Fig. 9. Unlike the situation in mitochondrial membranes, where $b$-type cytochromes $\left(b_{566}\right.$ and $\left.b_{561}\right)$ together with cytochrome $c_{1}$ have been shown to be similarly oriented to the terminal oxidase (Erecińska et al., 1978), the E. coli cytochrome(s) is aligned in the membrane with its $g_{\mathrm{z}}$ axis at $45^{\circ}$ to the membrane normal. The functional significance of these orientations must await information relating the principal $g$ axes to the geometry of the enzyme. It may be expected, however, that proteins with generally similar function and composition in various organisms will tend to have similar geometries.

Finally, although we have not explicitly studied the orientations of these cytochromes in membranes prepared from cells at discrete stages of the cell cycle, one conclusion can be drawn. The high degree of orientation observed in membranes prepared from an exponentially growing culture (containing cells at all cycle stages), especially for the low-spin component, must reflect the fact that the cytochrome orientation is invariant during the major portion of the cell cycle. Further work is required to relate on a time axis the syntheses of the various cytochromes, their incorporation (and perhaps subsequent orientation) in the membrane, and the acquisition of their functional roles.

R.K.P. thanks the Wellcome Trust for a Wellcome Research Travel Grant and the Science Research Council for grant GR/A/2252.5. This work was further supported by National Science Foundation grant PCM 78-16799 and National Institutes of Health grants GM 12202 and GM 25052. We are grateful to Dr W. John Ingledew for his critical reading of the manuscript.

\section{REFERENCES}

Altendorf, K. H. \& Staehelin, L. A. (1974). Orientation of membrane vesicles from Escherichia coli as detected by freeze-cleave electron microscopy. Journal of Bacteriology 117, 888-899.

Blasie, J. K., ERecińska, M., Samuels, S. \& Leigh, J. S. (1978). The structure of a cytochrome oxidase-lipid model membrane. Biochimica et biophysica acta 501, 33-52.

Blum, H., Harmon, H. J., Leigh, J. S., Salerno,
J. C. \& Chance, B. (1978a). The orientation of a heme of cytochrome $c$ oxidase in submitochondrial particles. Biochimica et biophysica acta 502, 1-10.

Blum, H., Salerno, J. C. \& Leigh, J. S. (1978b). A model for the simulation of the E.P.R. spectra of chromophores in partially oriented membrane multilayers. Journal of Magnetic Resonance 30, 385-391. 
Blum, H., Chance, B. \& Litchfield, W. J. (1978c). Effect of $\mathrm{pH}$ on bovine liver catalase as determined by electron paramagnetic resonance. Biochimica et biophysica acta 534, 317-321.

Blum, H., Chance, B., Gunson, D. E. \& LitchFIELD, W. J. $(1978 d)$. Electron paramagnetic resonance spectra of myeloperoxidase in polymorphonuclear leukocytes. FEBS Letters 86, $37-41$.

BlumberG, W. E. (1967). The EPR of high spin $\mathrm{Fe}^{3+}$ in rhombic fields. In Magnetic Resonance in Biological Systems, pp. 119-133. Edited by A. Ehrenberg, B. G. Malström \& T. Vänngard. Oxford: Pergamon Press.

Castor, L. N. \& ChanCE, B. (1959). Photochemical determinations of the oxidases of bacteria. Journal of Biological Chemistry 234, 1587-1592.

Crane, F. L., GlenN, J. L. \& Green, D. E. (1956). Studies on the electron transfer system. IV. The electron transfer particle. Biochimica et biophysica acta 22, 475-487.

Downie, J. A. \& Cox, G. B. (1978). Sequence of $b$ cytochromes relative to ubiquinone in the electron transport chain of Escherichia coli. Journal of Bacteriology 133, 477-484.

Erecińska, M., Blasie, J. K. \& Wilson, D. F. (1977). Orientation of the hemes of cytochrome $c$ oxidase and cytochrome $c$ in mitochondria. FEBS Letters 76, 235-239.

Erecińska, M., Wilson, D. F. \& Blasie, J. K. (1978). Studies on the orientations of the mitochondrial redox carriers. II. Orientation of the mitochondrial chromophores with respect to the plane of the membrane in hydrated, oriented mitochondrial multilayers. Biochimica et biophysica acta 501, 63-71.

Erecińska, M., Wilson, D. F. \& Blasie, J. K. (1979). Studies of the orientation of the mitochondrial redox carriers. III. Orientation of the $g_{\mathrm{x}}$ and $g_{\mathrm{y}}$ axes of the hemes of cytochrome oxidase with respect to the plane of the membrane in oriented membrane multilayers. Biochimica et biophysica acta 545, 352-364.

GARLAND, P. B. (1979). Vectorial oxidoreductions of bacterial respiratory chains. XIth International Congress of Biochemistry, Toronto, Canada, 1979, Abstract O6-7-Smp 4.

Garland, P. B., Davison, M. T. \& Moore, C. H. (1979). Rotational mobility of membrane-bound cytochrome $o$ of Escherichia coli and cytochrome $a_{1}$ of Thiobacillus ferro-oxidans. Biochemical Society Transactions 7, 1112-1114.

HADDock, B. A. \& Jones, C. W. (1977). Bacterial respiration. Bacteriological Reviews 41, 47-99.

Haddock, B. A., Downie, J. A. \& Garland, P. B. (1976). Kinetic characterization of the membranebound cytochromes of Escherichia coli grown under a variety of conditions by using a stoppedflow dual-wavelength spectrophotometer. Biochemical Journal 154, 285-294.

Hendler, R. W., Towne, D. W. \& Schrager, R. I. (1975). Redox properties of $b$-type cytochromes in Escherichia coli and rat liver mitochondria and techniques for their analysis. Biochimica et biophysica acta 376, 42-62.
Junge, W. \& Devault, D. (1975). Symmetry, orientation and rotational mobility in the $a_{3}$ haem of cytochrome $c$ oxidase in the inner membrane of mitochondria. Biochimica et biophysica acta 408, 200-214.

KABACK, H. R. (1971). Bacterial membranes. Methods in Enzymology 22, 99-120.

Knowles, P. F., Marsh, D. \& Rattle, H. W. E. (1976). Magnetic Resonance of Biomolecules, pp. 168-207. London: John Wiley.

Peisach, J., Blumberg, W. E., Ogawa, S., RachMILEWITZ, E. A. \& OLtzik, R. (1971). The effects of protein conformation on the heme symmetry in high spin ferric heme proteins as studied by electron paramagnetic resonance. Journal of Biological Chemistry 246, 3342-3355.

Poole, R. K. \& Haddock, B. A. (1974). Energylinked reduction of nicotinamide-adenine dinucleotide in membranes derived from normal and various respiratory-deficient mutant strains of Escherichia coli K12. Biochemical Journal 144, 77-85.

Poole, R. K. \& HaDDock, B. A. (1975). Effects of sulphate-limited growth in continuous culture on the electron-transport chain and energy conservation in Escherichia coli K12. Biochemical Journal 152, 537-546.

Poole, R. K., Waring, A. J. \& Chance, B. (1979a). Evidence for a functional oxygen-bound intermediate in the reaction of Escherichia coli cytochrome $o$ with oxygen. FEBS Letters 101, 56-58.

Poole, R. K., Waring, A. J. \& Chance, B. $(1979 b)$. The reaction of cytochrome $o$ in Escherichia coli with oxygen. Low temperature kinetic and spectral properties. Biochemical Journal 184, 379-389.

PudeK, M. R. \& BRAGG, P. D. (1976). Redox potentials of the cytochromes in the respiratory chain of aerobically-grown Escherichia coli. Archives of Biochemistry and Biophysics 174, 546552.

ReID, G. A. \& INGLEDEW, W. J. (1979). Characterization and phenotypic control of the cytochrome content of Escherichia coli. Biochemical Journal 182, 465-472.

Salerno, J. C., Blum, H. \& Ohnishi, T. (1979). The orientation of iron-sulfur clusters and a spincoupled ubiquinone pair in the mitochondrial membrane. Biochimica et biophysica acta 547, 270-281.

SHIPP, W. S. (1972). Cytochromes of Escherichia coli. Archives of Biochemistry and Biophysics 150, 459-472.

Tiede, D. M., Leigh, J. S. \& Dutton, P. L. (1978). Structural organization of the Chromatium vinosum reaction center associated $c$-cytochromes. Biochimica et biophysica acta 503, 524-544.

VAN Gelder, B. F., ORME-Johnson, W. H., HANSEN, R. E. \& BEINERT, H. (1967). Electron paramagnetic resonance of haem at intermediate oxidation states of cytochrome $c$ oxidase. Proceedings of the National Academy of Sciences of the United States of America 58, 1073-1079. 